\title{
Article
}

\section{Local Dynamics of Logistic Equation with Delay and Diffusion}

\author{
Sergey Kashchenko (1)
}

check for

updates

Citation: Kashchenko, S. Local Dynamics of Logistic Equation with Delay and Diffusion. Mathematics 2021, 9, 1566. https://doi.org/ 10.3390/math9131566

Academic Editors: Juan Ramón Torregrosa Sánchez, Alicia Cordero Barbero and Juan Carlos Cortés López

Received: 8 May 2021

Accepted: 1 July 2021

Published: 3 July 2021

Publisher's Note: MDPI stays neutral with regard to jurisdictional claims in published maps and institutional affiliations.

Copyright: (C) 2021 by the author. Licensee MDPI, Basel, Switzerland. This article is an open access article distributed under the terms and conditions of the Creative Commons Attribution (CC BY) license (https:// creativecommons.org/licenses/by/ $4.0 /)$.
Centre of Integrable Systems, P. G. Demidov State University, 150003 Yaroslavl, Russia; kasch@uniyar.ac.ru

\begin{abstract}
The behavior of all the solutions of the logistic equation with delay and diffusion in a sufficiently small positive neighborhood of the equilibrium state is studied. It is assumed that the Andronov-Hopf bifurcation conditions are met for the coefficients of the problem. Small perturbations of all coefficients are considered, including the delay coefficient and the coefficients of the boundary conditions. The conditions are studied when these perturbations depend on the spatial variable and when they are time-periodic functions. Equations on the central manifold are constructed as the main results. Their nonlocal dynamics determines the behavior of all the solutions of the original boundary value problem in a sufficiently small neighborhood of the equilibrium state. The ability to control the dynamics of the original problem using the phase change in the perturbing force is set. The numerical and analytical results regarding the dynamics of the system with parametric perturbation are obtained. The asymptotic formulas for the solutions of the original boundary value problem are given.
\end{abstract}

Keywords: logistic equation; diffusion; dynamics; stability; bifurcations; asymptotics; edge conditions; periodic solutions

\section{Introduction}

The logistic equation with delay

$$
\dot{u}=r[1-a u(t-T)] u
$$

is the natural generalization of the classical logistic equation. For example, it describes well the dynamics of biological population level changes [1-4]. All parameters in (1) are positive, and only non-negative solutions are considered, i.e., $u(t) \geq 0$. The parameter $r$ is called the Malthusian coefficient, $T$ is the delay time (for example, the age of individuals puberty). The parameter $a$ characterizes the resistance of the external environment. The literature on the study of the Equation (1) is extensive (see, for example, Refs. [5-9]).

We note that the zero solution in (1) is unstable, and the positive equilibrium state $u_{0}=a^{-1}$ is asymptotically stable under the condition $0<r T \leq \frac{\pi}{2}$. It was shown in [5] that the equilibrium state is globally stable for $0<r T<\frac{3}{2}$, i.e., each solution with a positive initial function tends to $u_{0}$ as $t \rightarrow \infty$. In the same paper [5], it was hypothesized that the global stability takes place in the wider range of parameters $0<r T \leq \frac{37}{24}$. This hypothesis is proved in $[7,8]$. The equilibrium state is unstable for $r T>\frac{\pi}{2}$, and (1) contains the stable cycle $u_{0}(t)$. The asymptotic behavior of the cycle has the form

$$
u_{0}(t)=a_{0}^{-1}\left(1+\sqrt{\epsilon}\left[\xi(\tau) \exp \left(i \frac{\pi}{2 T_{0}} t\right)+\bar{\xi}(\tau) \exp \left(-i \frac{\pi}{2 T_{0}} t\right)\right]\right)+O(\epsilon)
$$

for $0<r T-\frac{\pi}{2} \ll 1$. The cycle $u_{0}(t)$ is relaxation for $r T \gg 1$. Its asymptotic behavior is given in [9]. We also note that the number of unstable cycles in (1) grows indefinitely as $r T \rightarrow \infty$.

We consider the logistic equation with delay and diffusion

$$
\frac{\partial u}{\partial t}=d \frac{\partial^{2} u}{\partial x^{2}}+r[1-a u(t-T, x)] u, \quad x \in[0,1]
$$


with the boundary conditions

$$
\left.\frac{\partial u}{\partial x}\right|_{x=0}=\left.\frac{\partial u}{\partial x}\right|_{x=1}=0 .
$$

We fix the space $\mathbf{C}_{[-T, 0]} \times \mathbf{W}_{2[0,1]}^{2}$ as the space of initial conditions that are the nonnegative functions $\phi(t, x)$ for $t \in[-T, 0], x \in[0,1]$ satisfying the condition (4). The boundary value problem (3) and (4) naturally generalizes the Equation (1) for the problems of spatial interaction within one population. The boundary value problems of this type have been studied by many authors (see, for example, Refs. [10-12]). A fairly complete overview is given in [10].

First, we assume that all the coefficients are independent of $t$ and $x$. The boundary value problem (3) and (4) linearized on $u_{0}$ has the form

$$
\frac{\partial v}{\partial t}=d \frac{\partial^{2} v}{\partial x^{2}}-r v(t-T, x),\left.\quad \frac{\partial v}{\partial x}\right|_{x=0}=\left.\frac{\partial v}{\partial x}\right|_{x=1}=0 .
$$

The location of the roots of the characteristic quasi-polinomial for (5)

$$
\lambda=-d(2 \pi k)^{2}-r \exp (-\lambda T) \quad(k=0, \pm 1, \pm 2, \ldots)
$$

is of great importance for the stability of the equilibrium state $u_{0}$ of the boundary value problem (3) and (4). All roots of (6) have negative real parts under the condition $0<r T<\frac{\pi}{2}$, and (6) has the root with a positive real part as $r T>\frac{\pi}{2}$. Thus, in the first case, the solution $u_{0}$ is asymptotically stable, and is unstable in the second case. We consider the critical case when the equality

$$
r_{0} T_{0}=\frac{\pi}{2}
$$

holds for $d>0, r=r_{0}$ and $T=T_{0}$. In this case, the Equation (6) has a pair of pure imaginary roots $\pm i \omega_{0}$ for $k=0$ where $\omega_{0}=2 \pi / T_{0}$, and all its other roots have negative real parts.

We present the observation of the boundary value problem (3) and (4) dynamics in the neighborhood of the equilibrium state $u_{0}$ for the coefficients $d$ and $a$, which are close to some values $d_{0}$ and $a_{0}$, and the parameters $r$ and $T$ close to $r_{0}$ and $T_{0}$ for which the equality (7) holds. Let

$$
d_{0}=d_{0}+\epsilon d_{1}, \quad a=a_{0}+\epsilon a_{1}, \quad r=r_{0}+\epsilon r_{1}, \quad T=T_{0}+\epsilon T_{1}
$$

where $\epsilon$ is the small positive parameter

$$
0<\epsilon \ll 1 .
$$

We note that the positive equilibrium state $u_{0}=u_{0}(\epsilon)$ has the form $u_{0}=$ $a_{0}^{-} 1\left(1-\epsilon a_{1} a_{0}^{-1}+\ldots\right)$.

Under the above conditions, the two-dimensional stable local invariant integral manifold $M(\epsilon)$ exists in some sufficiently small and $\epsilon$-independent neighborhood of the equilibrium state $u_{0}(\epsilon)[13,14]$. On it, the boundary value problem (3) and (4) can be represented in the form

$$
\frac{d \xi}{d \tau}=\lambda_{1} \xi+\sigma \xi|\xi|^{2}
$$

up to terms of the order $O\left(\epsilon^{2}\right)$ where $\tau=\epsilon t$, and the equalities 


$$
\begin{aligned}
\lambda_{1} & =\left(1+\frac{\pi^{2}}{4}\right)^{-1}\left[\left(\frac{\pi}{2}+i\right) r_{1}+\lambda_{0}^{2} T_{1}\left(1-i \frac{\pi}{2}\right)\right] \\
\sigma & =-\lambda_{0}[3 \pi-2+i(\pi+6)]\left(10\left(1+\frac{4}{\pi^{2}}\right)\right)^{-1}
\end{aligned}
$$

are met for the coeffiicients $\lambda_{1}$ and $\sigma$.

The boundary value problem (3) and (4) solutions $u(t, \epsilon)$ are related with the (10) solutions by the asymptotic equality

$$
u(t, \epsilon)=u_{0}(\epsilon)+\epsilon^{1 / 2}\left(\xi(\tau) \exp \left(i \frac{\pi}{2 T_{0}} t\right)+\bar{\xi}(\tau) \exp \left(-i \frac{\pi}{2 T_{0}} t\right)\right)+O(\epsilon) .
$$

We note that $\Re \sigma<0$. Therefore, there is a stable cycle $\xi_{0}(\tau)=\xi_{0} \exp \left(i \phi_{0} \tau\right)$ for all $\Re \lambda_{1}>0$ in (10). Hence, in (3) and (4), there is the same stable cycle as in (1)

$$
\begin{aligned}
u_{0}(t, \epsilon)=u_{0}(\epsilon)+\sqrt{\epsilon}\left[\xi_{0}(\tau(1+O(\epsilon))) \exp \left(i \frac{2 \pi}{T_{0}} t\right)\right. & \left.+\bar{\xi}_{0}(\tau(1+O(\epsilon))) \exp \left(-i \frac{2 \pi}{T_{0}} t\right)\right] \\
& +\epsilon u_{2}(t, \tau)+\epsilon^{3 / 2} u_{3}(t, \tau)+\ldots
\end{aligned}
$$

where $\xi_{0}(\tau)=\xi_{0} \exp \left(i \phi_{0} \tau\right), \xi_{0}=\left[10\left(\frac{\pi}{2} \lambda_{1}+\lambda_{0}^{2} T_{1}\right)(3 \pi-2)^{-1}\right]^{1 / 2}, \phi_{0}=\Im \alpha_{1}+\xi_{0}^{2} \Im d$.

The above results are well known [15-17]. They describe the Andronov-Hopf bifurcation in regard to the boundary value problem (3) and (4).

In this paper, the Andronov-Hopf bifurcation is studied in more complex situations. In Sections 2-4, we suppose that the coefficients in (3) and (4) are close to the constants and depend on the spatial variable.

$$
d=d_{0}+\epsilon d_{1}(x), \quad a=1+\epsilon a_{1}(x), \quad r=r_{0}+\epsilon r_{1}(x), \quad T=T_{0}+\epsilon T_{1}(x), \quad r_{0} T_{0}=\frac{\pi}{2}
$$

where all the functions are sufficiently smooth.

The Andronov-Hopf bifurcation under the conditions (13) is considered in Section 2. In Section 3, the perturbations also concern the boundary conditions. The Dirichlet boundary conditions are considered in Section 4 . Section 5 considers non-autonomous perturbations. In particular, the problem of parametric resonance is considered.

\section{Spatially Inhomogeneous Perturbations of Boundary Value Problem (3) and (4) Coefficients}

We consider the boundary value problem (3) and (4) under the conditions (13).

\subsection{Andronov-Hopf Bifurcation under Neumann Type Boundary Conditions}

First, we find the asymptotic behavior of the positive equilibrium state $K(x, \epsilon)$ as $\epsilon \rightarrow 0$. The equation

$$
d K^{\prime \prime}+r[1-a K] K=0,\left.\quad K^{\prime}\right|_{x=0}=\left.K^{\prime}\right|_{x=1}=0
$$

holds for it.

Since the value $K \equiv 1$ is a simple root of the nonlinear function in (14) for $\epsilon=0$, it is natural to look for $K(x, \epsilon)$ in the form

$$
K(x, \epsilon)=1+\epsilon K_{1}(x)+\epsilon^{2} K_{2}(x)+\ldots
$$

We substitute (15) into (3) and (4) and collect the coefficients at the same powers of $\epsilon$. We successively find $K_{1}(x), K_{2}(x), \ldots$ from the resulting relations. So, collecting the coefficients at the first power of $\epsilon$, we obtain 


$$
d_{0} K_{1}^{\prime \prime}-r_{0}\left(K_{1}+a_{1}\right)=0,\left.\quad K_{1}^{\prime}\right|_{0}=\left.K_{1}^{\prime}\right|_{1}=0 .
$$

From here,

$$
K_{1}(x)=\kappa^{-1}\left[\int_{0}^{1} \sinh \left(\kappa(1-s) a_{1}(s)\right) d s-(\sinh \kappa)^{-1} \cosh \kappa x \cdot \int_{0}^{1} \cosh \left(\kappa(1-s) a_{1}(s)\right) d s\right]
$$

where $\kappa=\left(r_{0} d_{0}^{-1}\right)^{1 / 2}$.

The following result holds.

Theorem 1. The boundary value problem (3) and (4) has the positive equilibrium state $K(x, \epsilon)$ for which the asymptotic equality (15) holds.

\subsection{Linear Analysis}

We linearize (3) and (4) at $K(x, \epsilon)$. As a result, we obtain the boundary value problem

$$
\begin{gathered}
\frac{\partial v}{\partial t}=d \frac{\partial^{2} v}{\partial x^{2}}+r(1-a K) v-r a K v(t-T, x), \\
\left.\frac{\partial v}{\partial x}\right|_{x=0}=\left.\frac{\partial v}{\partial x}\right|_{x=1}=0 .
\end{gathered}
$$

Setting $v=\exp (\lambda t) w(x)$, we obtain the characteristic equation

$$
d w^{\prime \prime}+\mu w=0,\left.\quad w^{\prime}\right|_{x=0}=\left.w^{\prime}\right|_{x=1}=0
$$

where

$$
\mu=\mu(x, \epsilon)=r(1-a K)-\lambda-r a K \exp (-\lambda T) .
$$

For $\epsilon=0$, we have the equation with the constant coefficients

$$
d_{0} w^{\prime \prime}+\mu^{0} w=0,\left.\quad w^{\prime}\right|_{x=0}=\left.w^{\prime}\right|_{x=1}=0
$$

where

$$
\mu^{0}=-\lambda-r_{0} \exp \left(-\lambda T_{0}\right)
$$

The equalities

$$
\mu_{n}^{0}=d_{0}(\pi n)^{2}, \quad w_{n}(x)=\cos \pi n x, \quad n=0,1,2, \ldots
$$

hold for the eigenvalues $\mu_{n}^{0}$ and the eigenfunctions $w_{n}(x)$ of the boundary value problem (20). Taking into consideration the relation (21), we obtain the following statement for the roots of the equations

$$
\mu_{n}^{0}=-\lambda-r_{0} \exp \left(-\lambda T_{0}\right) \quad(n=0,1, \ldots) .
$$

Lemma 1. For $n=0$, Equation (22) has two roots on the imaginary axis $\lambda_{0}^{ \pm}= \pm i \pi\left(2 T_{0}\right)^{-1}$, and all other roots of this equation and all the roots of all the equations (22) have negative real parts for $n \neq 0$.

We note that the eigenfunction $w_{0}(x) \equiv 1$ corresponds to the roots $\lambda_{0}^{ \pm}$.

We find the asymptotic behavior of those two complex conjugated roots $\lambda^{ \pm}(\epsilon)$ that tend to $\pm i \pi\left(2 T_{0}\right)^{-1}$ as $\epsilon \rightarrow 0$. Let 


$$
\begin{gathered}
\lambda=\lambda^{+}(\epsilon)=i \pi\left(2 T_{0}\right)^{-1}+\epsilon \lambda_{1}+\epsilon^{2} \lambda_{2}+\ldots, \\
w_{0}(x, \epsilon)=1+\epsilon w_{1}(x)+\epsilon^{2} w_{2}(x)+\ldots
\end{gathered}
$$

in (19).

Collecting the coefficients at the first power of $\epsilon$, we obtain the equation

$$
d_{0} w_{1}^{\prime \prime}-r_{0} w_{1}=f(x),\left.\quad w_{1}^{\prime}\right|_{x=0}=\left.w_{1}^{\prime}\right|_{x=1}=0
$$

where

$$
f(x)=\lambda_{1}\left(1+i \frac{\pi}{2}\right)+r_{0}(1-i)\left(a_{1}(x)+K_{1}(x)\right)-i r_{1}(x)+\frac{\pi}{2 T_{0}} r_{0} T_{1}(x) .
$$

The boundary value problem (25) is solvable if and only if the equality

$$
\int_{0}^{1} f(x) d x=0
$$

holds. Taking into consideration (26), we come to the conclusion that

$$
\begin{gathered}
\lambda_{1}=\left(1+i \frac{\pi}{2}\right)^{-1} \int_{0}^{1}\left[i r_{1}(x)-r_{0}(1-i)\left(a_{1}(x)+K_{1}(x)\right)-\frac{\pi}{2 T_{0}} r_{0} T_{1}(x)\right] d x, \\
w_{1}(x)=d_{0}^{-1} \int_{0}^{x} \int_{0}^{s} f(\tau) d \tau d s .
\end{gathered}
$$

Collecting the coefficients at $\epsilon^{2}$ (in (19) with the equalities (23) and (24)), we obtain $\lambda_{2}, \ldots$ The formula (27) can be simplified. From (16), it follows that $\int_{0}^{1}\left(a_{1}(x)+K_{1}(x)\right) d x=0$; therefore, $\lambda_{1}=\left(1+i \frac{\pi}{2}\right)^{-1} \int_{0}^{1}\left[i r_{1}(x)-\frac{\pi}{2 T_{0}} r_{0} T_{1}(x)\right] d x$.

2.3. Boundary Value Problem (3) and (4) Dynamics in Neighborhood of Equilibrium $K(x, \epsilon)$ under Conditions (13)

The characteristic Equation (19) has two roots close to the imaginary axis under these conditions, and all of its remaining roots lie to the left of the imaginary axis and are separated from this axis as $\epsilon \rightarrow 0$. Then, in the small neighborhood of $K(x, \epsilon)$, the local stable two-dimensional integral manifold exists (see, for example, Refs. $[13,14]$ ) where the boundary value problem (3) and (4) can be written as one complex first-order equation

$$
\frac{d \xi}{d \tau}=\beta \xi+\gamma \xi|\xi|^{2}
$$

to within $O\left(\epsilon^{2}\right)$. Here, $\tau=\epsilon t$ is a "slow" time, and the "amplitude" $\xi(\tau)$ is related to the solutions of (3) and (4) by the asymptotic equality

$$
\begin{aligned}
& u(t, x, \epsilon)=K(x, \epsilon) \\
& +\epsilon^{1 / 2}\left(\left(1+\epsilon w_{1}(x)\right) \xi(\tau) \exp \left(i \frac{\pi}{2 T_{0}} t\right)+\left(1+\epsilon w_{1}(x)\right) \bar{\xi}(\tau) \exp \left(-i \frac{\pi}{2 T_{0}} t\right)\right) \\
& +\epsilon\left(u_{20}|\xi|^{2}+u_{21} \xi^{2} \exp \left(i \frac{\pi}{T_{0}} t\right)+\bar{u}_{21} \bar{\xi}^{2} \exp \left(-i \frac{\pi}{T_{0}} t\right)\right)+\epsilon^{-3 / 2} u_{3}(t, \tau, x)+\ldots
\end{aligned}
$$


where $u_{3}$ is periodic with respect to $t$.

We substitute (29) in (3). Then, collecting the coefficients at the same powers of $\epsilon$, we obtain the correct equality for $\epsilon^{1 / 2}$. At the second step, equating the coefficients of $\epsilon^{1}$ on the left and right sides of the formal equality, we obtain the expressions for $u_{20}$ and $u_{21}$ :

$$
u_{20}=0, \quad u_{21}=A \xi^{2} \exp (2 i \sigma t)+\bar{A} \bar{\xi}^{2} \exp (-2 i \sigma t), \quad A=\frac{2-i}{5} .
$$

At the next step, we arrive at the equation in $u_{3}(t, \tau, x)$. From the condition of its solvability in the class of $\pi / 2 T_{0}$-periodic with respect to $t$ functions, we obtain the equality (28) for $\xi(\tau)$, where $\beta=\lambda_{1}$, and $\gamma=\sigma$ as in the Equation (10).

Let us summarize what has been said.

Theorem 2. Let $\Re \lambda_{1}<0$. Then, for all sufficiently small $\epsilon$, all the solutions from some $\epsilon$ independent neighborhood of the equilibrium $K(x, \epsilon)$ tend to it as $t \rightarrow \infty$. If $\Re \lambda_{1}>0$, then there is the stable cycle $u_{0}(t, x, \epsilon)$ in the neighborhood of $K(x, \epsilon)$ for which

$$
\begin{aligned}
& u_{0}(t, x, \epsilon)=K(x, \epsilon) \\
& +\epsilon^{1 / 2}\left(\xi_{0}(\tau)\left(1+\epsilon w_{1}(x)\right) \exp \left(i \pi\left(2 T_{0}\right)^{-1} \tilde{t}\right)+\bar{\xi}_{0}(\tau)\left(1+\epsilon w_{1}(x)\right) \exp \left(-i \pi\left(2 T_{0}\right)^{-1} \tilde{t}\right)\right) \\
& \quad+\epsilon\left(u_{20}\left|\xi_{0}\right|^{2}+u_{21} \xi_{0}^{2}(\tau) \exp \left(i \pi\left(T_{0}\right)^{-1} \tilde{t}\right)+u_{21} \bar{\xi}_{0}(\tau) \exp \left(-i \pi\left(T_{0}\right)^{-1} \tilde{t}\right)\right)+O\left(\epsilon^{3 / 2}\right)
\end{aligned}
$$

where $\tilde{t}=\left(1+o\left(\epsilon^{2}\right)\right) t$.

We note that the coefficients $d_{1}(x)$ and $a_{1}(x)$ do not affect the values of the coefficients in the Equation (28). The coefficients $r_{1}(x)$ and $T_{1}(x)$ determine the value of $\beta=\lambda_{1}$ only through their mean values on the segment $x \in[0,1]$.

\section{Bifurcations under Disturbances in Boundary Conditions}

Instead of the Neumann boundary conditions (4), we consider the "perturbed" boundary conditions

$$
\left.\frac{\partial u}{\partial x}\right|_{x=0}=\epsilon \alpha_{1}\left(\left.u\right|_{x=0}-\gamma_{1}\right),\left.\quad \frac{\partial u}{\partial x}\right|_{x=1}=\epsilon \alpha_{2}\left(\left.u\right|_{x=1}-\gamma_{2}\right)
$$

for the Equation (3). Here, $\alpha_{1}, \alpha_{2}, \gamma_{1}, \gamma_{2}$ are some fixed constants. For simplicity, all the coefficients in (3) are assumed constant for simplicity: $d=d_{0}, a=1, r=r_{0}, T=T_{0}$ and $r_{0} T_{0}=\frac{\pi}{2}$.

First, we find the asymptotic behavior of the positive equilibrium $K(x, \epsilon)$ of the boundary value problem (3) and (31). For this, we put $K(x, \epsilon)=1+\epsilon K_{1}(x)+\ldots$. We substitute this expression in (3) and (31). Then, we obtain the boundary value problem

$$
d_{0} K_{1}^{\prime \prime}-r_{0} K_{1}=0,\left.\quad K_{1}^{\prime}\right|_{x=0}=\tilde{\alpha}_{1},\left.\quad K_{1}^{\prime}\right|_{x=1}=\tilde{\alpha}_{2}, \quad \tilde{\alpha}_{j}=\alpha_{j}\left(1-\gamma_{j}\right)
$$

for $K_{1}(x)$. We find from here that

$$
K_{1}(x)=\left(\tilde{\alpha}_{2}-\tilde{\alpha}_{1} \cosh \kappa\right)(\kappa \sinh \kappa)^{-1} \cosh (\kappa x)+\tilde{\alpha}_{1} \kappa^{-1} \sinh \kappa x
$$

where $\kappa=\left(r_{0} d_{0}^{-1}\right)^{1 / 2}$.

The characteristic equation of the boundary value problem linearized on $K(x, \epsilon)$ has a pair of pure imaginary roots $\pm i \pi\left(2 T_{0}\right)^{-1}$ as $\epsilon=0$. All its other roots have negative real parts.

We find the asymptotic behavior of those two complex conjugate roots $\lambda^{ \pm}(\epsilon)$ of this characteristic equation 0 which tend to $\lambda_{0}^{ \pm}$as $\epsilon \rightarrow 0$. Let 0

$$
\lambda^{+}(\epsilon)=\lambda_{0}^{+}+\epsilon \lambda_{10}+\epsilon^{2} \lambda_{20}+\ldots
$$


We look for the eigenfunction corresponding to the root $\lambda^{+}(\epsilon)$ in the form

$$
w(x, \epsilon)=1+\epsilon w_{1}(x)+\epsilon^{2} w_{2}(x)+\ldots
$$

We substitute (33) and (34) into Equation (19) with the boundary conditions

$$
\left.\frac{\partial w}{\partial x}\right|_{x=0}=\left.\epsilon \alpha_{1} w\right|_{x=0},\left.\quad \frac{\partial w}{\partial x}\right|_{x=1}=\left.\epsilon \alpha_{2} w\right|_{x=1} .
$$

Collecting the coefficients at the first power of $\epsilon$ in the resulting formal identity, we obtain the boundary value problem (19) for $w_{1}(x)$ in which

$$
\mu=-\lambda_{10}\left(1+i \frac{\pi}{2}\right)-r_{0}(1-i) K_{1}
$$

From this, we find that

$$
\begin{aligned}
& \lambda_{10}=\left(1+i \frac{\pi}{2}\right)^{-1} r_{0}(i-1) \int_{0}^{1} K_{1}(x) d x+d_{0}\left(\alpha_{2}-\alpha_{1}\right) \\
& =\left(1+i \frac{\pi}{2}\right)^{-1} d_{0}(i-1)\left(\tilde{\alpha}_{2}-\tilde{\alpha}_{1}\right)+d_{0}\left(\alpha_{2}-\alpha_{1}\right) .
\end{aligned}
$$

At the final stage, we use the line of reasoning from the previous section. We substitute the formal series (29) in (3) and (31) and collect the coefficients at the same powers of $\epsilon$. For $\epsilon^{1 / 2}$, we obtain the correct equality. At the next step, we find the expressions for $u_{20}$ and $u_{21}$ which are also defined by the equalities (30). Then, we obtain the equation for $u_{3}(t, \tau, x)$. From its solvability condition in the class of $4 T_{0}$-periodic with respect to $t$ functions, we obtain the Equation (28) where

$$
\beta=\lambda_{10}, \quad \gamma=\sigma
$$

It remains to repeat Theorem 2 with $\lambda_{1}$ replaced by $\lambda_{10}$ as a final statement.

We note that the parameters of the boundary conditions determine the value of $\lambda_{10}$, and all bifurcation effects depend only on the relation $\tilde{\alpha}_{2}-\tilde{\alpha}_{1}$, i.e., on $\alpha_{2}-\alpha_{1}+\gamma_{1}-\gamma_{2}$.

\section{Andronov-Hopf Bifurcation in Case of Dirichlet Boundary Conditions}

We consider the bifurcations in the equilibrium neighborhood of the boundary value problem

$$
\begin{gathered}
\frac{\partial u}{\partial t}=d \frac{\partial^{2} u}{\partial x^{2}}-r u(t-T, x)[1+u], \\
\left.u\right|_{x=0}=0,\left.\quad u\right|_{x=1}=0 .
\end{gathered}
$$

Here, we assume that all the coefficients are close to the constant values

$$
d=d_{0}+\epsilon d_{1}(x), \quad r=r_{0}+\epsilon r_{1}(x), \quad T=T_{0}+\epsilon T_{1}(x) .
$$

The values of $d_{0}, r_{0}$ and $T_{0}$ are determined below from the condition for the existance of the critical case in the zero equilibrium (38) and (39) stability problem. The characteristic equation of the linearized at zero boundary value problem has the form

$$
d w^{\prime \prime}+\mu w=0, \quad w(0)=w(1)=0
$$

where $\mu=-\lambda-r \exp (-\lambda T)$. The smallest value $\mu_{0}$ of the boundary value problem (40) is equal to $\pi^{2} d_{0}$ as $\epsilon=0$, and the corresponding eigenfunction is $\sin \pi x$.

The bifurcation values $d_{0}, r_{0}$ and $T_{0}$ are found from the condition $\lambda=i \omega_{0}$, i.e., from the equation

$$
\pi^{2} d_{0}=-i \omega_{0}-r_{0} \exp \left(-i \omega_{0} T_{0}\right)
$$

From this, we obtain

$$
r_{0} \cos \omega_{0} T_{0}=-\pi^{2} d, \quad r_{0} \sin \omega_{0} T_{0}=\omega_{0}
$$


The value $\omega_{0}$ is determined from the following equation,

$$
\tan \omega_{0} T_{0}=-\omega_{0}\left(\pi^{2} d_{0}\right)^{-1},
$$

and the value $r_{0}$ is determined from the equality

$$
r_{0}^{2}=\omega_{0}^{2}+\left(\pi^{2} d_{0}\right)^{2} .
$$

Below, we fix the positive constants $d_{0}$ and $T_{0}$ arbitrarily and denote by $r_{0}$, the expression (42).

For small values of $\epsilon$, the eigenvalue $\lambda(\epsilon)$, which is close to $i \omega_{0}$ as $\epsilon \rightarrow 0$, and the corresponding eigenfunction $w(x, \epsilon)$ are sought in the form

$$
\lambda(\epsilon)=i \omega_{0}+\epsilon \lambda_{1}+\ldots \quad w(x, \epsilon)=\sin \pi x+\epsilon w_{1}(x)+\ldots
$$

We substitute (43) in (38) and (39) and collect the terms at the first power of $\epsilon$ to find $\lambda_{1}$ and $w_{1}(x)$. Then,

$$
d_{0} w_{1}^{\prime \prime}+\mu_{0} w_{1}=f(x) \sin \pi x
$$

where

$$
f(x)=\left[-\lambda_{1}\left(1+T_{0} r_{0} \exp \left(-i \omega_{0} T_{0}\right)\right)+\left[r_{1}(x)-i \omega_{0} r_{0} T_{1}(x)\right] \exp \left(-i \omega_{0} T_{0}\right)-d_{1} \pi^{2}\right] \sin (\pi x) .
$$

The boundary value problem (44) is solvable if and only if the equality

$$
\int_{0}^{1} f(x) \sin (\pi x) d x=0 .
$$

holds. Given (45) in it, we find the value of $\lambda_{1}$ :

$$
\begin{aligned}
& \lambda_{1}=\left(2\left(1+\pi^{2} d_{0}+i \omega_{0} T_{0}\right)\right)^{-1}\left[\exp \left(-i \omega_{0} T_{0}\right)\right. \\
& \left.\times\left(i \omega_{0} T_{0} \int_{0}^{1} T_{1} \sin ^{2}(\pi x) d x-\int_{0}^{1} r_{1}(x) \sin ^{2}(\pi x) d s\right)-\frac{\pi^{2}}{2} \int d_{1} d x\right] .
\end{aligned}
$$

After that, we define $w_{1}(x)$ :

$$
w_{1}(x)=\pi^{-1} \int_{0}^{x} \sin \pi(x-s) f(s) \sin \pi s d s .
$$

We use the above formal description when investigating the solutions of (38) and (39) from a sufficiently small neighborhood of the zero equilibrium. The series

$$
\begin{aligned}
& u(t, x, \epsilon)=\epsilon^{1 / 2}\left(\xi(\tau)\left(\sin \pi x+\epsilon w_{1}(x)+\ldots\right) \exp \left(i \omega_{0} t\right)\right. \\
& \left.+\bar{\xi}\left(\sin \pi x+\epsilon w_{1}(x)+\ldots\right) \exp \left(-i \omega_{0} t\right)\right) \\
& +\epsilon\left(u_{20}(x)|\xi(\tau)|^{2}+u_{21}(x) \xi^{2}(\tau) \exp \left(2 i \omega_{0} t\right)+\bar{u}_{21} \bar{\xi}^{2}(\tau) \exp \left(-2 i \omega_{0} t\right)\right) \\
& +\epsilon^{3 / 2}\left(u_{31}(x) \xi^{3}(\tau) \exp \left(3 i \omega_{0} t\right)+\overline{c c}+u_{32}(x) \xi(\tau)|\xi(\tau)|^{2} \exp \left(i \omega_{0} t\right)+\overline{c c}\right. \\
& \left.+u_{30}(x) \xi(\tau) \exp \left(i \omega_{0} t\right)+\overline{c c}\right)+\ldots
\end{aligned}
$$

is an analogue of the asymptotic series (29). The expression complex conjugate to the previous term is denoted by $\overline{c c}$ here. We substitute (48) into (38) and (39). Performing standard actions, we obtain the equalities for $u_{20}(x)$ and $u_{21}(x)$ at the second step: 


$$
\begin{aligned}
& u_{20}(x)=r_{0}\left(\exp \left(i \omega_{0} T_{0}\right)+\exp \left(-i \omega_{0} T_{0}\right)\right) \kappa^{-1} \\
& \times\left(\int_{0}^{x} \sinh \kappa(x-s) \sin ^{2} s d s-(\sinh \kappa)^{-1} \sinh \kappa x \cdot \int_{0}^{1} \sinh \kappa(1-s) \sin ^{2} s d s\right), \\
& u_{21}(x)=\kappa_{1}^{-1} r_{0} \exp \left(-i \omega_{0} T_{0}\right) \\
& \times\left(\int_{0}^{x} \sinh \left(\kappa_{1}(x-s)\right) \sin ^{2} s d s-\left(\sinh \kappa_{1}\right)^{-1} \sinh \kappa_{1} x \cdot \int_{0}^{1} \sinh \left(\kappa_{1}(1-s) \sin ^{2} s d s\right)\right), \\
& \kappa=\left(r_{0} d^{-1}\right)^{1 / 2}, \quad \kappa_{1}=\left(-\left(2 i \omega_{0}+r_{0} \exp \left(-2 i \omega_{0} T_{0}\right)\right) d_{0}^{-1}\right)^{1 / 2} .
\end{aligned}
$$

Finally, we obtain the equations for $u_{30}(x)$ and $u_{31}(x)$ at the next step. From their solvability condition, we obtain the Equation (10) in which the formula (46) holds for $\lambda_{1}$, and the formula

$$
\begin{aligned}
\sigma=-\int_{0}^{1}\left[2\left(1+\pi^{2} d_{0}+i \omega_{0} T_{0}\right)\right]^{-1} r_{0}[ & u_{20}(s)\left(1+\exp \left(-i \omega_{0} T_{0}\right)\right) \\
& \left.+u_{21}(s)\left(\exp \left(i \omega_{0} T_{0}\right)+\exp \left(-2 i \omega_{0} T_{0}\right)\right)\right] \sin s d s
\end{aligned}
$$

holds for $\sigma$.

The main statement repeats the formulation of Theorem 2 in this case too.

Remark 1. Unlike the results of the previous sections, the role of the coefficient $d_{1}(x)$ increases for the boundary conditions (39). Its mean value is included in the formula (46) for the coefficient $\lambda_{1}$.

Remark 2. The next statement follows from the results presented above related to the linearized problem for (38) and (39). For any $T_{0}>0$ and $r_{0}>\frac{\pi}{2 T_{0}}$, we can choose such $d_{0}$ that the AndronovHopf bifurcation condition holds: the characteristic equation has a pair of roots on the imaginary axis for $\epsilon=0$, and all its other roots have negative real parts. In particular, this means that the increase in the diffusion coefficient leads to zero equilibrium stability in (38) and (39).

\section{Bifurcations under Time-Periodic Perturbations}

In order to illustrate more clearly the new features that arise in the case of time-periodic perturbations in the logistic equation with delay and diffusion, we investigate the simplest cases. We present the main general results in Section 5.1. The obtained results are applied to solve the problem of parametric resonance in Section 5.2. A more complicated problem of parametric resonance with a two-frequency perturbation is studied in Section 5.3.

\subsection{Main Results}

We consider the equation

$$
\frac{\partial u}{\partial t}=d_{0} \frac{\partial^{2} u}{\partial x^{2}}-r_{0} u(t-1, x)[1+u]
$$

with the periodic boundary conditions

$$
\left.\frac{\partial u}{\partial x}\right|_{x=0}=\left.\epsilon \alpha_{1}(t) u\right|_{x=0},\left.\quad \frac{\partial u}{\partial x}\right|_{x=1}=\left.\epsilon \alpha_{2}(t) u\right|_{x=1} .
$$

Here, $r_{0}=\frac{\pi}{2}, d_{0}>0$ and the functions $\alpha_{j}(t)(j=1,2)$ are periodic with the period $h$. In addition, $\alpha_{j}(t)=\alpha_{j 0}+\alpha_{j 1}(t)$ and 


$$
M\left(\alpha_{j}(t)\right)=\frac{1}{h} \int_{0}^{h} \alpha_{j}(t) d t=0 .
$$

For $\epsilon=0$, the critical case occurs in the problem of zero equilibrium stability in (49) and (50): the corresponding characteristic equation

$$
\lambda=-d_{0} k^{2}-r_{0} \exp (-\lambda) \quad(k=0, \pm 1, \pm 2, \ldots)
$$

has two roots $\lambda_{0}^{ \pm}= \pm i \frac{\pi}{2}$ on the imaginary axis, and all its other roots have a negative real part. According to the above technique for studying the solutions from zero neighborhood of the boundary value problem (49) and (50), we introduce into consideration the formal series

$$
u=\epsilon^{1 / 2}\left(\xi(\tau) \exp \left(i \frac{\pi}{2} t\right)+\overline{c c}\right)+\epsilon u_{2}(t, \tau)+\epsilon^{3 / 2} u_{3}(t, \tau, x)+\ldots
$$

Here, $\tau=\epsilon t$ is a "slow" time, and the dependence on the argument $t$ is 4-periodic. We substitute (52) in (49) and (50) and collect the coefficients at the same powers of $\epsilon$ in the resulting formal identity. We obtain the correct equality for $\epsilon^{1 / 2}$. For the first power of $\epsilon$, we obtain the equation for $u_{2}(t, \tau)$, and the formula for this function coincides with (30). Collecting the coefficients of $\epsilon^{3 / 2}$ at the third step, we obtain the equation for $u_{3}$ :

$$
\begin{gathered}
\frac{\partial u_{3}}{\partial t}=d_{0} \frac{\partial^{2} u_{3}}{\partial x^{2}}-r_{0} u_{3}(t-1, \tau, x)+f(t, \tau), \\
\left.\frac{\partial u_{3}}{\partial x}\right|_{x=0}=\alpha_{1}(t)\left(\xi(\tau) \exp \left(i \frac{\pi}{2} t\right)+\bar{\xi}(\tau) \exp \left(-i \frac{\pi}{2} t\right)\right), \\
\left.\frac{\partial u_{3}}{\partial x}\right|_{x=1}=\alpha_{2}(t)\left(\xi(\tau) \exp \left(i \frac{\pi}{2} t\right)+\bar{\xi}(\tau) \exp \left(-i \frac{\pi}{2} t\right)\right)
\end{gathered}
$$

where

$$
\begin{aligned}
f(t, \tau)=(i-1) u_{21} & \exp \left(i \frac{\pi}{2} t\right) \xi|\xi|^{2}+\overline{c c} \\
& -(1+i) u_{21} \exp \left(i \frac{3 \pi}{2} t\right) \xi^{3}+\overline{c c}-\left(1+i \frac{\pi}{2}\right) \frac{\partial \xi}{\partial \tau} \cdot \exp \left(i \frac{\pi}{2} t\right)+\overline{c c}
\end{aligned}
$$

We use the criterion for the boundary value problem (53)-(55) solvability in the class of 4 -periodic with respect to $t$ functions. For this, it is necessary and sufficient that the equality

$$
\left(1+i \frac{\pi}{2}\right) \frac{\partial \xi}{\partial \tau}=d_{0} M\left(\alpha_{2}(t)-\alpha_{1}(t)\right) \xi+d_{0} M\left(\left(\alpha_{2}(t)-\alpha_{1}(t)\right) \exp (-i \pi t)\right) \bar{\xi}+\sigma \xi|\xi|^{2}
$$

holds. Here, the value $\sigma$ is the same as above.

We note that the Equation (56) takes the simplest form

$$
\frac{d \xi}{d \tau}=A \xi+\sigma \xi|\xi|^{2}
$$

when the functions $\alpha_{1,2}(t)$ do not contain harmonics with the doubled frequency of the natural oscillations $\exp \left( \pm 2 i \frac{\pi}{2} t\right)$. Here, $A=\left(1+i \frac{\pi}{2}\right)^{-1} d_{0}\left(\alpha_{20}-\alpha_{10}\right)$. The solutions of (57) can be obtained in an explicit form.

Under the condition

$$
\Re A=d_{0}\left(1+\frac{\pi^{2}}{4}\right)^{-1}\left(\alpha_{20}-\alpha_{10}\right)<0
$$


all the solutions of (57) tend to zero as $t \rightarrow \infty$. Hence, we conclude that for sufficiently small $\epsilon$, all the solutions of (49) and (50) from a sufficiently small and independent of $\epsilon$ neighborhood of the zero equilibrium also tend to zero as $t \rightarrow \infty$.

If $\Re A>0$, then (57) contains the stable cycle

$$
\xi_{0}(\tau)=\xi_{0} \exp i \phi_{0} \tau, \quad \xi_{0}=\left[(-\Re A)(\Re \sigma)^{-1}\right]^{1 / 2}, \quad \phi_{0}=\Im A+(\Im \sigma)\left(\xi_{0}\right)^{2} .
$$

This means that the zero equilibrium of the boundary value problem (49) and (50) is unstable and the stable cycle

$$
\begin{aligned}
u_{0}(t, \epsilon, x)=\epsilon^{1 / 2}\left[\xi_{0}(\tau(1+O(\epsilon)))\right. & \exp \left(i \frac{\pi}{2} t\left(1+O\left(\epsilon^{3 / 2}\right)\right)\right) \\
& \left.+\bar{\zeta}_{0}(\tau(1+O(\epsilon))) \exp \left(-i \frac{\pi}{2} t\left(1+O\left(\epsilon^{3 / 2}\right)\right)\right)\right]+O(\epsilon)
\end{aligned}
$$

exists.

Then, we consider the case when the functions $\alpha_{1,2}(t)$ have harmonics with the frequencies $\pm i \pi t$, i.e.,

$$
\alpha_{j 1}(t)=b_{j} \exp (i \pi t)+\bar{b}_{j} \exp (-i \pi t)+b_{j}(t)
$$

where $b_{j}(t)$ do not contain harmonics with zero and $\pm i \pi$ frequencies. The Equation (56) takes the form

$$
\frac{d \xi}{d \tau}=A \xi+B \bar{\zeta}+\sigma \xi|\xi|^{2}
$$

where $B=\left(1+i \frac{\pi}{2}\right)^{-1} d_{0}\left(b_{2}-b_{1}\right)$.

\subsection{Problem of Parametric Resonance}

In this section, the results obtained in Section 5.1 are used to study the problem of parametric resonance. Let the formulas

$$
\alpha_{j}(t)=\alpha_{j 0}+b_{j} \cos \omega t
$$

hold for the periodic functions $\alpha_{1,2}(t)$.

We assume here that

$$
\alpha_{20}-\alpha_{10}<0,
$$

i.e., the zero solution in (49) and (50) is asymptotically stable in the absence of nonautonomous components in (51), and for all sufficiently small $\epsilon$, all the solutions of (49) and (50) from a sufficiently small and $\epsilon$ independent neighborhood of zero equilibrium tend to zero as $t \rightarrow \infty$. The same conclusion is valid under the conditions when $b_{j} \neq 0$ and the inequality

$$
\omega \neq \pi
$$

holds for the arbitrary parameter $\omega$.

We consider the case of $\omega=\pi$. The condition of zero solution stability in (49) and (50) is defined by the zero solution stability in (58). The corresponding criterion is formulated in terms of the matrix

$$
C=\left(\begin{array}{cc}
\Re A+\Re B, & -\Im A+\Im B \\
\Im A+\Im B, & \Re A-\Re B
\end{array}\right)
$$

eigenvalues. By virtue of (60), we have the equality $\operatorname{tr} C=2 \Re A=2 d_{0}\left(1+\frac{\pi^{2}}{4}\right)^{-1}\left(\alpha_{20}-\right.$ $\left.\alpha_{10}\right)<0$. Therefore, both eigenvalues of the matrix $C$ have negative real parts under the condition 


$$
\Delta=\operatorname{det} C=|A|^{2}-|B|^{2}=d_{0}\left(1+\frac{\pi^{2}}{4}\right)^{-2}\left(\left(\alpha_{20}-\alpha_{10}\right)^{2}-\left(b_{2}-b_{1}\right)^{2}\right)>0 .
$$

Thus, the zero equilibrium in (58) and in (49) and (50) is asymptotically stable.

If $\Delta<0$, then the zero equilibrium in (58) and in (49) and (50) is unstable (for small $\epsilon$ ). We assume below that

$$
\Delta<0 .
$$

We investigate the problem of parametric resonance, i.e., the problem of the parameter $\omega$ entire set selection for which the zero solution of (49) and (50) is unstable. For this, we arbitrarily fix the parameter $z$ and put

$$
\omega=\pi(1+\epsilon z)
$$

in (49) and (50).

To ensure that the oscillation frequency does not depend on $\epsilon$ under the boundary conditions (50), we change the time

$$
(1+\epsilon z) t=t_{1} .
$$

As a result, we obtain the boundary value problem

$$
\begin{gathered}
\frac{\partial u}{\partial t_{1}}=(1+\epsilon z)^{-1}\left[\frac{\partial^{2} u}{\partial x^{2}}-r_{0} u\left(t_{1}-(1+\epsilon z)\right)(1+u)\right] \\
\left.\frac{\partial u}{\partial x}\right|_{x=0}=\left.\epsilon\left(\alpha_{10}+b_{1} \cos \pi t_{1}\right) u\right|_{x=0},\left.\quad \frac{\partial u}{\partial x}\right|_{x=1}=\left.\epsilon\left(\alpha_{20}+b_{2} \cos \pi t\right) u\right|_{x=1} .
\end{gathered}
$$

To study the solutions of this boundary value problem, we use the above algorithm. Let

$$
u=\epsilon^{1 / 2}\left(\xi(\tau) \exp \left(i \frac{\pi}{2} t\right)+\overline{c c}\right)+\epsilon u_{2}(t, \tau, x)+\epsilon^{3 / 2} u_{3}(t, \tau, x)+\ldots
$$

in (65) and (66) where $\tau=\epsilon t$ and $u_{j}(t, \tau, x)$ are 4-periodic with respect to $t$. Again, we obtain the formula (30) for $u_{2}(t, \tau)$, and from the equation solvability condition with respect to $u_{3}$, we get the final equation for determining $\xi$

$$
\frac{\partial \xi}{\partial \tau}=\left(-i \frac{\pi}{2} z+A\right) \xi+B \bar{\xi}+\sigma \xi|\xi|^{2}
$$

where the values $A, B$ and $\sigma$ are the same as in (58). The stability of the zero solution is determined by the eigenvalues of the matrix

$$
C_{1}=C+\frac{\pi}{2} z\left(\begin{array}{cc}
0 & 1 \\
-1 & 0
\end{array}\right)
$$

We note that $\operatorname{tr} C_{1}=\operatorname{tr} C<0$ and $\operatorname{det} C_{1}=\frac{\pi^{2}}{4} z^{2}+m z+\Delta$ where $m=d_{0}\left(\alpha_{20}-\right.$ $\left.\alpha_{10}\right)\left(2\left(1+\frac{\pi^{2}}{4}\right)\right)^{-1}$. Let us formulate a simple statement about the roots of the equation $\operatorname{det} C_{1}=0$.

Lemma 2. The inequality

$$
\operatorname{det} C_{1}<0
$$

holds under the conditions

$$
z^{+}<z<z^{-}
$$

where $z^{ \pm}=\frac{2}{\pi^{2}}\left(-m \pm \sqrt{m^{2}-\pi^{2} \Delta}\right)$. 
The inequalities (70) define the set of those values of the frequency $\omega$ of (63) for which the zero solution in (65) and (66) (for small $\epsilon$ ) and in (68) is unstable. It is at these frequencies that parametric resonance is observed. The dynamics of the (68) solutions have been studied by many authors (see, for example, Refs. [18,19]).

\subsection{Example. Dynamics at Same Frequencies and Different Phases of External Influences}

As an example, we consider a slightly different from (59) situation when

$$
\alpha_{1}(t)=\alpha_{10}+b_{1} \cos \omega t, \quad \alpha_{2}(t)=\alpha_{20}+b_{2} \cos (\omega t+\phi)
$$

where $\phi \in[0,2 \pi]$ and the equality

$$
\omega=\pi(1+\epsilon w)
$$

holds.

Performing the above sequence of actions in order to determine the local dynamics of the boundary value problem (49) and (50), we obtain the normal form, i.e., the equation for the unknown "amplitude" $\xi(\tau)(\tau=\epsilon t)$ :

$$
\frac{d \xi}{d \tau}=\alpha \xi+\beta(\phi) \exp (i \pi w \tau) \bar{\xi}+\sigma \xi|\xi|^{2}
$$

in which the coefficients $\alpha$ and $\sigma$ are the same as in (68), and

$$
\beta(\phi)=\left(1+i \frac{\pi}{2}\right)^{-1} d_{0}\left(b_{20} \exp (i \phi)-b_{10}\right) .
$$

Replacing $\xi=v \exp \left(i \frac{\pi}{2} w \tau\right)$, we obtain the equation

$$
\frac{d v}{d \tau}=\alpha_{0} v+\beta(\phi) \bar{v}+\sigma v|v|^{2}
$$

and $\alpha_{0}=\alpha-\frac{1}{2} i \pi w$. The stability of the zero solution in (73) is determined by the sign of the expression $\rho(\phi)$ where

$$
\rho(\phi)=d_{0}^{2}\left[\left(\alpha_{10}-\alpha_{20}\right)^{2}-\left(b_{10}-b_{20}\right)^{2}-4 b_{10} b_{20} \sin ^{2} \frac{\phi}{2}\right] .
$$

The zero solution in (73) is asymptotically stable under the condition $\rho(\phi)>0$, and it is unstable when $\rho(\phi)<0$. It remains to note that the function $\rho(\phi)$ is alternating in some range of parameters variation in (73) as $\phi \in[0,2 \pi]$. Thus, the phase shift in (71) can significantly change the dynamic behavior of the original boundary value problem (49) and (50).

\subsection{Parametric Resonance for Two-Frequency Perturbation}

In contrast to the periodic perturbations in (59), here we assume that the frequencies of each of the function $\alpha_{1}(t)$ and $\alpha_{2}(t)$ are different:

$$
\alpha_{1}(t)=\alpha_{10}+b_{1} \cos w_{1} t, \quad \alpha_{2}(t)=\alpha_{20}+b_{2} \cos w_{2} t .
$$

In addition, we assume that the inequality (60) holds and each of the values $\omega_{1}$ and $\omega_{2}$ is close to the doubled natural oscillations frequency, i.e.,

$$
\omega_{j}=\pi\left(1+\epsilon w_{j}\right) \quad(j=1,2) .
$$

We note that the paper [20] investigated the problem of the parametric resonance for a two-frequency perturbation in the linear equation with delay. In the paper [21], this issue was studied in a nonlinear statement of the problem for the logistic equation with delay where the coefficients $r$ and $T$ were perturbed by two-frequency actions.

The algorithmic approach here repeats the constructions of the previous section. We consider the formal asymptotic series (67) and substitute it in (49) and (50) taking into 
account the equalities (74) and (75). We collect the coefficients at the same powers of $\epsilon$ in the resulting formal identity. At the second step, we obtain the formulas (30) here too. From the condition of solvability of the equation with respect to $u_{3}$, we obtain the equation for $\xi(\tau)$ determining:

$$
\frac{d \xi}{d \tau}=\alpha \xi+B(\tau) \bar{\xi}+\sigma \xi|\xi|^{2}
$$

Here, $\alpha=\left(1+i \frac{\pi}{2}\right)^{-1} d_{0}\left(\alpha_{20}-\alpha_{10}\right), B(\tau)=\left(1+i \frac{\pi}{2}\right)^{-1} d_{0}\left(b_{20} \exp \left(i \pi w_{2} \tau\right)-b_{10} \exp \right.$ $\left.\left(i \pi w_{1} \tau\right)\right)$. The parameter $\sigma$ is the same as in (58).

Let, for definiteness, $w_{2}>w_{1}$. We perform the following changes in (76): $\xi=$ $v \exp \left(i \frac{\pi}{2} w_{1} \tau\right), \alpha_{0}=\alpha-i w_{1}, \alpha_{j}=\left(1+i \frac{\pi}{2}\right)^{-1} d_{0} b_{j}(j=1,2), \pi\left(w_{2}-w_{1}\right)=\mu$ and $\tau_{1}=\mu \tau$. As a result, we obtain the equation with $2 \pi$-periodic coefficients

$$
\mu \frac{d v}{d \tau_{1}}=\alpha_{0} v+\left(\alpha_{2} \exp \left(i \tau_{1}\right)-\alpha_{1}\right) \bar{v}+\sigma v|v|^{2} .
$$

We recall that $\Re \alpha_{0}<0, \Re \sigma<0$.

We investigate the dynamics of the Equation (77) for various values of the parameters $\mu, w_{1}, \alpha_{1}, \alpha_{2}$. It should be noted that the "crude" periodic solution of (77) corresponds to the two-dimensional torus of the same stability.

The Equation (77) is well studied in the case of the parametric resonance with a single-frequency perturbation, i.e., for $\alpha_{2}=0$ (see, for example, Refs. [18,19]).

The stability of the linear part of the Equation (77) was studied in [20]. Below, are the relevant results. First, we represent the linear part of (77) in the real form

$$
\begin{gathered}
\mu \frac{d \omega}{d \tau_{1}}=B\left(\tau_{1}\right) \omega, \quad \omega=\left(\begin{array}{c}
\Re v \\
\Im v
\end{array}\right), \\
B\left(\tau_{1}\right)=\left(\begin{array}{cc}
\Re \alpha_{0}+B_{1}\left(\tau_{1}\right) & -\Im \alpha_{0}+B_{2}\left(\tau_{1}\right) \\
\Im \alpha_{0}+B_{2}\left(\tau_{1}\right) & \Re \alpha_{0}-B_{1}\left(\tau_{1}\right)
\end{array}\right)
\end{gathered}
$$

where $B_{1}\left(\tau_{1}\right)=\Re \alpha_{1}+\Re \alpha_{2} \cos \tau_{1}-\Im \alpha_{2} \sin \tau_{1}, B_{2}\left(\tau_{1}\right)=\Im \alpha_{1}+\Re \alpha_{2} \sin \tau_{1}+\Im \alpha_{2} \cos \tau_{1}$.

For sufficiently large $\mu$, we apply the well-known averaging principle [22]. The stability properties are then determined by the averaged equation

$$
\mu \frac{d \omega}{d \tau_{1}}=B_{0} \omega, \quad B_{0}=\left(\begin{array}{cc}
\Re \alpha_{0}+\Re \alpha_{1} & -\Im \alpha_{0}+\Im \alpha_{1} \\
\Im \alpha_{0}+\Im \alpha_{1} & \Re \alpha_{0}-\Re \alpha_{1}
\end{array}\right) .
$$

Thus, the case of large values of $\mu$ is reduced to the case of the parametric resonance with a single-frequency perturbation.

We suppose now that

$$
0<\mu \ll 1 .
$$

Then, according to the papers $[23,24]$ results, the stability of the zero solution of the system (78) is closely related to the behavior of the function

$$
\rho\left(\tau_{1}\right)=\operatorname{det}\left(B\left(\tau_{1}\right)-\Re \alpha_{0} \cdot E\right) .
$$

Three options are distinguished in the system (78) study depending on whether the function $\rho\left(\tau_{1}\right)$ is positive, negative, or alternating. Taking into account the form of the matrix $B\left(\tau_{1}\right)$ in $(78)$, we have

$$
\rho\left(\tau_{1}\right)=\left(\Im \alpha_{0}\right)^{2}-\left(B_{1}^{2}\left(\tau_{1}\right)+B_{2}^{2}\left(\tau_{1}\right)\right) .
$$

Thus, any of these conditions can be fulfilled by appropriate change of $\Im \alpha_{0}$. Following [21], we describe the dynamics of the system (78). As it turned out, there are three following options for the behavior of this system solutions: 
1. Let $\rho(\tau)>0$ for all $\tau>0$. Then, there exists $\mu_{0}>0$ such that for $0<\mu \leq \mu_{0}$, the zero solution of the system (78) is asymptotically stable.

2. Let $\rho(\tau)<0$ for all $\tau>0$. Then, there exists $\mu_{0}>0$ such that for $0<\mu \leq \mu_{0}$, the zero solution of (78) is asymptotically stable (unstable) if

$$
\frac{1}{2 \pi} \int_{0}^{2 \pi} \sqrt{-\rho(\tau)} d \tau+\Re \alpha_{0}<0 \quad(>0) .
$$

3. Finally, in the case of the alternating function $\rho(\tau)$, the problem (78) is a system with turning points. Moreover, if the condition

$$
\frac{1}{2 \pi} \int_{0}^{2 \pi} \sqrt{\frac{|\rho(\tau)|-\rho(\tau)}{2}} d \tau>\Re \alpha_{0}
$$

holds then the stability and instability of the system (78) solutions alternate infinitely for $\mu \rightarrow 0$. (We note that if the inequality is replaced by the opposite in (82), then the zero solution is asymptotically stable for sufficiently small $\mu$ ).

The properties listed above of the system (78) solutions allow us to draw the conclusions about the local stability or instability of the zero solution of the nonlinear Equation (77) for sufficiently large or small enough $\mu$. The phase rearrangements of the Equation (77) are not asymptotically analizable at conditionally "average" values of the parameter $\mu$. In this regard, it is appropriate to apply numerical methods. The acceptability of their application for finding the stable modes of the Equation (77) is due, in particular, to its dissipativity $(\Re \sigma<0)$. The latter property makes it possible to choose the initial conditions only from some neighborhood of the zero point of the phase plane. The detailed numerical study of the Equation (77) is given in [21]. The following conclusions were formulated on its basis.

- In the case of the parametric resonance with a two-frequency perturbation, the dynamics is fundamentally more complicated than in the case of a single-frequency perturbation. Cycles and regions (in the parameter space) of the irregular behavior of the solutions appear much more complex in their form.

- If $\rho\left(\tau_{1}\right)<0$ i.e., the asymptotic stability of the zero solution exists, no non-stationary steady-state solutions were found.

- The dynamic behavior is relatively simple for the sufficiently large $\mu$. We have the complicated relaxation stable cycles for the sufficiently small $\mu$. They are either symmetric or two stable symmetric cycles coexisting with respect to each other.

- Irregular oscillations are specific for the "average" values of the parameter $\mu$.

- Irregular oscillations in (77) are realized in the relatively narrow ranges of $\mu$ variation. In this case, the phase rearrangements are related with the symmetry-loss bifurcations, with the cascades of period-doubling bifurcations and with the bifurcations of separatrix splitting.

\section{Delayed Perturbation Influence on Boundary Value Problem (49) and (50) Dynamic Behavior}

We suppose that the small perturbation containing the delay is added to the right-hand side of the Equation (49)

$$
\begin{gathered}
\frac{\partial u}{\partial t}=d \frac{\partial^{2} u}{\partial x^{2}}-r u(t-1, x)[1+u]+\epsilon\left(f_{1} u+f_{2} u(t-h, x)\right), \\
\left.\frac{\partial u}{\partial x}\right|_{x=0}=\left.\frac{\partial u}{\partial x}\right|_{x=1}=0 .
\end{gathered}
$$

Here, $d>0$ and $r=\frac{\pi}{2}$. Thus, the Andronov-Hopf bifurcation occurs. For fixed $h$, $f_{1}$ and $f_{2}$, the problem of local dynamics of (83) and (84) in the neighborhood of the zero equilibrium is solved in the same was as in Sections 2 and 3. We obtain the normal form 


$$
\frac{d \xi}{d \tau}=\left(1+i \frac{\pi}{2}\right)^{-1}\left[f_{1}+f_{2} \exp \left(-i \frac{\pi}{2} h\right)\right] \xi+\sigma \xi|\xi|^{2}
$$

with the same coefficient $\sigma$ as in (10).

Under the condition that the delay value $h$ (83) is large enough, the problem becomes much more complicated. Let us show this below.

Let the equality

$$
h=h_{1} \epsilon^{-1}
$$

be met for some fixed value $h_{1}>0$.

We consider the characteristic equation for the linearized at zero boundary value problem (83) and (84):

$$
\lambda=-\frac{\pi}{2} \exp (-\lambda)+\epsilon f_{1}+\epsilon f_{2} \exp \left(-\lambda h_{1} \epsilon^{-1}\right) .
$$

Under the condition (86), infinitely many of its roots tend to the imaginary axis as $\epsilon \rightarrow 0$.

In the case (86), the algorithm for the local dynamics study is the same as above $[25,26]$. We consider the formal series (11) for $T_{0}=1$. Then, as a result of standard actions, we obtain the normal form similar to (10), which is the equation with delay

$$
\frac{d \xi}{d \tau}=\left(1+i \frac{\pi}{2}\right)^{-1}\left[f_{1} \xi+f_{2} \exp (i \phi) \xi\left(\tau-h_{1}\right)\right]=\sigma \xi|\xi|^{2} .
$$

The main result is that according to the asymptotic formula (11), the nonlocal dynamics of the Equation (88) determines the local behavior of the boundary value problem (83) and (84) solution. An important distinction from (10) is not only in the appearance of the containing delay term. The expression (88) includes the parameter $\exp (i \phi)$, which infinitely runs over all values on the unit circle on the complex plane as $\epsilon \rightarrow 0$. Therefore, we can only assert that the determined for some $\phi=\phi_{0}$, some steady state in (88) corresponds to the solution of the boundary value problem (83) and (84) with asymptotic behavior (11) for the sufficiently small values of $\epsilon=\epsilon_{n}$ where

$$
\epsilon_{n}=\pi h_{1}\left[2\left(\phi_{0}+2 \pi n\right)\right]^{-1} .
$$

The dynamic behavior of (88) may differ for different values of $\phi$. This means that an infinite process of forward and reverse bifurcations can occur in (83) and (84) as $\epsilon \rightarrow 0$. We note that interesting results were obtained on the Equation (88) dynamics under the condition $h_{1} \gg 1$ in $[25,27]$.

\section{Conclusions}

The questions regarding the behavior of all the solutions of the logistic equation with delay and diffusion in a small neighborhood of a positive equilibrium are considered. It is assumed that the most common and significant conditions are fulfilled when the critical Andronov-Hopf case is realized in the problem of the equilibrium stability. All coefficients differ from the constant values by some rather small function of the spatial or time variable. In particular, we note that the perturbations of both the delay coefficient and the boundary conditions coefficients are considered.

The normal forms are constructed. They are the special complex ordinary differential first-order equations on the central manifold, the nonlocal dynamics that determines the behavior of all the solutions of the original boudary value problem in a small neighborhood of the equilibrium. The asymptotic formulas that couple the solutions of the parabolic boundary value problem with the normal form solutions are presented.

In the case when the small time-periodic perturbation with the frequency close to the double that of natural oscillations affects the equation coefficients, the equation becomes more complicated on the central manifold. The problem of parametric resonance is 
considered as an application. In particular, it is shown that under certain conditions, the dynamics of the original boundary value problem can be controlled by means of a phase shift in a small periodic perturbation. The most complicated results concern the problem of parametric resonance with a two-frequency perturbation. The conditions under which the local dynamics of the problem under consideration can be complex and irregular are revealed with the help of numerical and analytical methods.

Funding: This research was funded by Ministry of Science and Higher Education of the Russian Federation (075-02-2020-1514).

Data Availability Statement: Not applicable.

Conflicts of Interest: The author declares no conflict of interest.

\section{References}

1. Murray, J.D. Mathematical Biology II, 3rd ed.; Number 18 in Interdisciplinary Applied Mathematics; Springer: New York, NY, USA, 2003. [CrossRef]

2. Okuba, A. Dynamical aspects of animal grouping: Swarms, schools, flocks, and herds. Adv. Biophys. 1986, 22, 1-94. [CrossRef]

3. Wu, J. Theory and Applications of Partial Functional Differential Equations; Number 119 in Applied Mathematical Sciences; Springer: New York, NY, USA, 1996. [CrossRef]

4. Kuang, Y. Delay Differential Equations: With Applications in Population Dynamics; Number 191 in Mathematics in Science and Engineering; Academic Press: Boston, MA, USA, 1993.

5. Wright, E.M. A non-linear difference-differential equation. J. Reine Angew. Math. 1955, 194, 66-87. [CrossRef]

6. Kakutani, S.; Markus, L. On the non-linear difference-differential equation $y^{\prime}(t)=[A-B y(t-\tau)] y(t)$. In Contributions to the Theory of Nonlinear Oscillations; Lefschetz, S., Ed.; Princeton University Press: Princeton, NJ, USA, 1958; Volume 4, pp. 1-18. [CrossRef]

7. Kaschenko, S.A.; Loginov, D.O. About Global Stable of Solutions of Logistic Equation with Delay. J. Phys. Conf. Ser. 2017, 937, 012019. [CrossRef]

8. Kashchenko, S.A.; Loginov, D.O. Estimation of the Region of Global Stability of the Equilibrium State of the Logistic Equation with Delay. Russ. Math. 2020, 64, 34-49. [CrossRef]

9. Kashchenko, S.A. Asymptotics of the Solutions of the Generalized Hutchinson Equation. Autom. Control. Comput. Sci. 2013, 47, 470-494. [CrossRef]

10. Gourley, S.A.; Sou, J.W.H.; Wu, J.H. Nonlocality of Reaction-Diffusion Equations Induced by Delay: Biological Modeling and Nonlinear Dynamics. J. Math. Sci. 2004, 124, 5119-5153. [CrossRef]

11. Gourley, S.A.; Britton, N.F. A predator-prey reaction-diffusion system with nonlocal effects. J. Math. Biol. 1996, 34, 297-333. [CrossRef]

12. Levin, S.A. Population models and community structure in heterogeneous environments. In Mathematical Ecology: An Introduction; Hallam, T.G., Levin, S.A., Eds.; Springer: Berlin/Heidelberg, Germany, 1986; pp. 295-320. [CrossRef]

13. Hale, J.K. Theory of Functional Differential Equations, 2nd ed.; Springer: New York, NY, USA, 1977.

14. Hartman, P. Ordinary Differential Equations; Wiley: New York, NY, USA, 1965.

15. Oster, G.; Guckenheimer, J. Bifurcation Phenomena in Population Models. In The Hopf Bifurcation and Its Applications; Number 19 in Applied Mathematical Sciences; Springer: New York, NY, USA, 1976; pp. 327-353. [CrossRef]

16. Kashchenko, S.A.; Loginov, D.O. Bifurcations due to the variation of boundary conditions in the logistic equation with delay and diffusion. Math. Notes 2019, 106, 136-141. [CrossRef]

17. So, J.; Wu, J.; Yang, Y. Numerical steady state and Hopf bifurcation analysis on the diffusive Nicholson's blowflies equation. Appl. Math. Comput. 2000, 111, 53-69. [CrossRef]

18. Bautin, N.N.; Leontovich, E.A. Methods and Rules for the Qualitative Study of Dynamical Systems on the Plane, 2nd ed.; Nauka: Moscow, Russia, 1990.

19. Guckenheimer, J.; Holmes, P. Nonlinear Oscillations, Dynamical Systems, and Bifurcations of Vector Fields; Number 42 in Applied Mathematical Sciences; Springer: New York, NY, USA, 1983. [CrossRef]

20. Kashchenko, S.A.; Kolesov, Y.S. Parametric resonance in systems with delay under a two-frequency perturbation. Sib. Math. J. 1980, 21, 231-235. [CrossRef]

21. Bykova, N.D.; Glyzin, S.D.; Kashchenko, S.A. Parametric Resonance in the Logistic Equation with Delay under a Two-Frequency Perturbation. Nonlinear Phenom. Complex Syst. 2016, 19, 80-87. [CrossRef]

22. Bogoliubov, N.N.; Mitropolsky, Y.A. Asymptotic Methods in the Theory of Non-Linear Oscillations; Number 10 in Russian Monographs and Texts on Advanced Mathematics and Physics; Hindustan Publ. Corp.: Delhi, India, 1961.

23. Kashchenko, S.A. Asymptotic Expansions of Eigenvalues of Periodic and Antiperiodic Boundary Value Problems for Singularly Perturbed Second-Order Differential Equation with Turning Points. Autom. Control. Comput. Sci. 2018, 52, 728-744. [CrossRef]

24. Kashchenko, S.A. Asymptotical Distributions of Eigenvalues of Periodic and Antiperiodic Boundary Value Problems for Second-Order Differential Equations. Autom. Control. Comput. Sci. 2018, 52, 797-809. [CrossRef] 
25. Kashchenko, S.A.; Loginov, D.O. Andronov-Hopf Bifurcation in Logistic Delay Equations with Diffusion and Rapidly Oscillating Coefficients. Math. Notes 2020, 108, 50-63. [CrossRef]

26. Kashchenko, S.A. Bifurcations in the Neighborhood of a Cycle under Small Perturbations with a Large Delay. Comput. Math. Math. Phys. 2000, 40, 659-668.

27. Kaschenko, S.A. Bifurcational Features in Systems of Nonlinear Parabolic Equations with Weak Diffusion. Int. J. Bifurc. Chaos Appl. Sci. Eng. 2005, 15, 3595-3606. [CrossRef] 